\title{
Computer Assisted Orthognathic Surgery
}

\author{
B. Mollard*, S. Lavallée* and G. Bettegat \\ *TIMC Laboratory - IAB, Faculté de Médecine de Grenoble \\ 38706 La Tronche, France \\ + Plastic and Maxillo-facial Surgery department \\ CHU A. Michallon - B.P. 21738043 Grenoble Cedex, France \\ Benoit.Mollardeimag.fr
}

\begin{abstract}
This paper presents a surgical simulator for orthognathic surgery based on the integration of dental models and 3D cephalometry. The objective of dental models integration is to make coherent informations gathered from different sources (occlusal analysis and CT scan), and for that purpose, a system using a 3D optical localizer is used. The 3D Cephalometry analysis is used for the detection of dysmorphosis and surgical planning. This cephalometry integrates the Inferrence process for improving the surgical system. Both elements of our simulator have been implemented and technically validated with success.
\end{abstract}

\section{Introduction}

Planning cranio-facial surgical procedures, particularly orthognathic surgery, requires integration of multiple and complex data gathered from different sources: clinical examination (anthropometry), orthodontic (dental models), radiological (cephalometry) and intra-operative data (constraints and position information). This heterogeneity makes the therapeutic decision difficult, particularly in asymmetrical dysmorphosys. This is the reason why several three-dimensional (3D) surgical analysis and simulation softwares and methods have been developed $[2,8,6,11,7]$.

This paper introduces a 3D cephalometric analysis system and a surgical simulator for orthognatic surgery developed by Bettega et al. [2]. Our simulator is based on the integration of dental models (section 2) and 3D cephalometry (section 3). We discuss the notion of Inferrence and its benefit in terms of improvement of the surgical system (section 4). Those concepts are illustrated on concrete and quantified data.

\section{Dental Models Integration}

The goal of dental model integration is to transpose the results of the occlusal analysis carried out on teetli plaster cast by the prosthesist into the 3D CT scan models visualized and manipulated by the surgeon. The objective is to make both informations coherent, for better accuracy and efficiency. For that purpose, we use a three-dimensional optical localizer (Optotrak, NorthernDigital, Toronto). First, two intercuspidation splints are constructed. These resine splints 
are modeled with the plaster casts, according to the usual procedure followed by the orthodontist and the prosthesist. The initial intercuspidation splint $\left(I C S_{i}\right)$ is used to materialize the mandible position in preoperative occlusion and to allow the ulterior fusion of the data given by the localizer and those extracted from the CT slices (Figure 1). During the acquisition of the patient CT-scan, ICS will guaranty that the preoperative occlusion is respected. This plate is also used to support a rigid body (infra-red emitting system) localized by Optotrak. The final intercuspidation splint $\left(I C S_{f}\right)$ corresponds to the occlusal correction. It is used to mobilise the plaster casts, which are manually displaced in order to engage the teeth in the desired position after the intervention. The final splint is built by the prosthesist using an articulation jaw and plaster casts, following a long-term orthodontic treatment which has displaced the teeth towards a desired position.

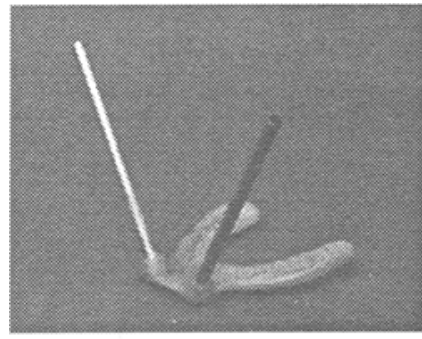

(a)

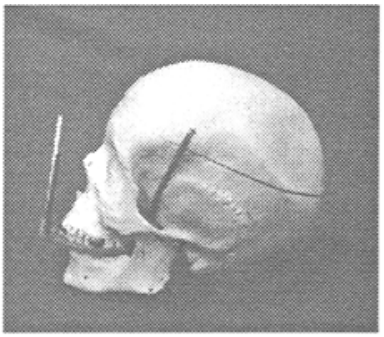

(b)

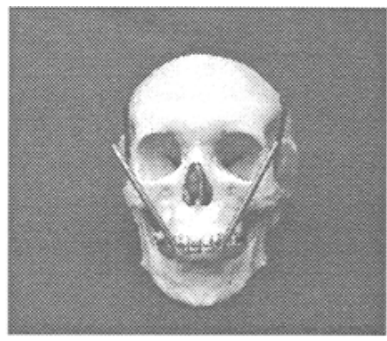

(c)

Fig. 1. Lateral(b) and facial(c) views of a dry skull equipped with $I C S_{i}$ (a). This splint is equipped with external landmarks made up of two aluminium tubes (implanted on the splint with a guide plate) easily identifiable in CT scan slices (without causing radiological artefacts) and constituting a 3D referential visible by Optotrak. These landmarks tubes allow matching the CT scan and the dental data with accuracy.

The change from the initial splint to the final one is represented by a displacement matrix $T_{1}$ (Figure $2(\mathrm{a})$ ). In other words, $T_{1}$ defines the relative position of maxilla in relation to the mandible after the intercuspidation splint has been changed. The scheme for accurate determination of $T_{1}$ consists of digitizing the position of infra-red emitting systems firmly fixed to plaster cast, first with $\mathbf{I} \mathbf{C} \mathbf{S}_{\mathbf{i}}$, then with $\mathbf{I C S}_{\mathbf{f}}$. The relative displacement given by $T 1$ has to be transferred into the tridimensional reconstruction of the CT-scan referential. This is done by the application of a registration algorithm between landmarks tubes digitized with Optotrak, and those tubes pointed on CT-scan slices (Figure 2 (b)). In order to evaluate the accuracy of the landmarks tube-based registration method, we have compared it with a 3D-3D rigid registration algorithm using octree-splines [9] (Figure 3 ). We have compared the rotation and translation components of both transformations: 


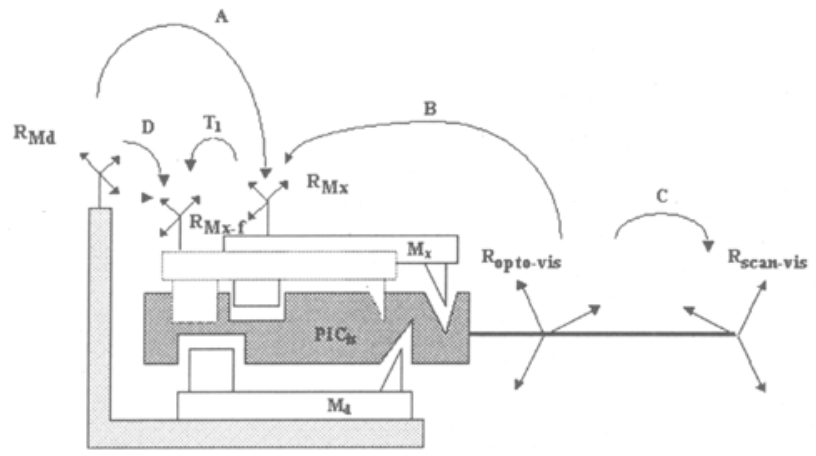

(a)

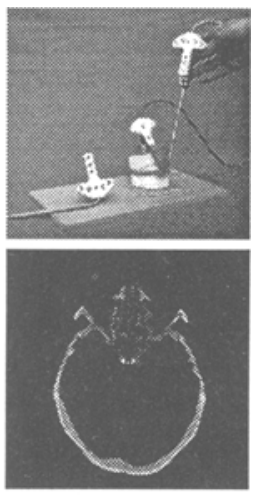

(b)

Fig. 2. (a) Identification of the different matrices - (b) Digitization of Landmarks Tubes: it consists of making a sensor equipped with a rigid body sliding and rotating into the tube. About one hundred points are digitized in this way and for each tube - Calculation of the line which goes through the longitudinal axis of each tube after manual localisation on each CT-scan slice of the center of both tube slices

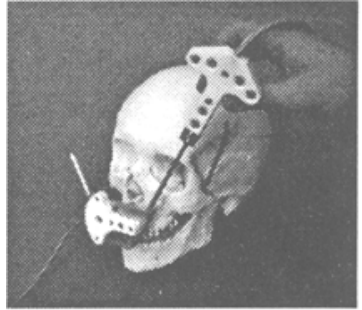

(a)

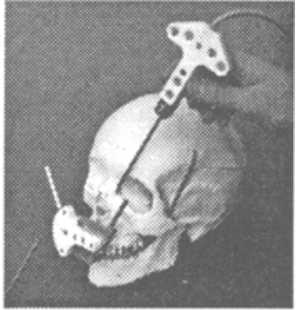

(b)

Fig. 3. (a) Evaluation of matrix $C$ using the tubes (b) Evaluation of matrix $C$ using the $3 \mathrm{D}-3 \mathrm{D}$ non-rigid registration algorithm: on the bone surface of a dry skull equipped with $I C S_{i}, 200$ points are digitized in $R_{\text {opto-vis }}$ referential. A 3D CT scan reconstruction of the bone surface is performed in $R_{\text {scan-vis }}$ referential. Therefore, the optical data (cloud of points) are matched with the C'T scan data (points extracted from the $3 \mathrm{D}$ reconstruction of the bone surface).

The rotation error is $0.93 \mathrm{dg}$. The translation error is $0.43 \mathrm{~mm}$.

Both transforms are obtained using very different data. Results show that they are probably both equally accurate. Those results are quite acceptable and validate our approach. With our system, it is possible to integrate the dental models in the $3 \mathrm{D}$ cephalometry and so, in the surgical simulator. 


\section{3D Cephalometry}

A 3D cephalometry software has been developped to generalize 2D Delaire Cephalometry [4] and take advantage of 3D capabilities to allow the integration of the problems of facial asymetry and occlusal plane horizontality into the profile analysis (Figure 4). It was implemented using Open-Inventor.
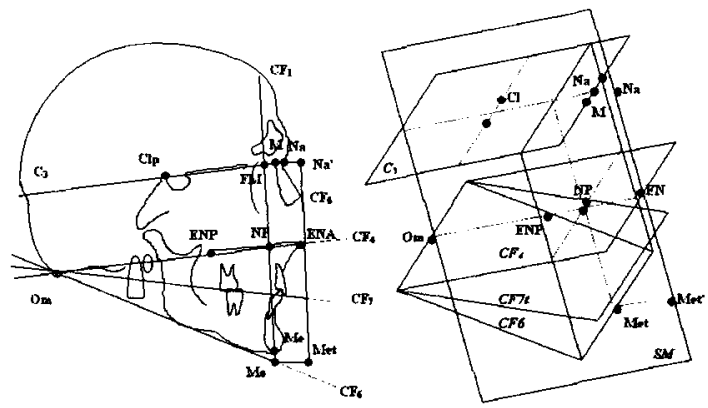

Fig. 4. 2D Representation of simplified Delaire analysis and 3D Representation of cephalometric points and planes

3D Reconstruction Modalities - The Marching Cubes method is used to reconstruct the skull from computer tomography [10]. The rendering quality is excellent but the number of triangles generated to reconstruct the bone surfaces is too huge to allow an interactive surgical simulation. We don't use compression algorithms to reduce the number of facets because the operator must have a sufficiently precise model to identify correctly the cephalometrical points without losing any detail. The chosen solution is to build an "hybrid" model of the skull (Figure 5).

3D Cephalometry - The 3D Cephalometrical analysis procedure is then divided in 3 steps: definition of sagittal median plane (Figure $6(\mathrm{a})$ ), determination of 10 cephalometrical anatomical landmarks (Figure 6 (b)) and the analysis itself, which is automatically calculated (Figure 6 (c)). Each step of the analysis is validated and if necessary manually corrected.

Virtual Osteotomies - The goal is to isolate on the 3D model a part of the maxilla and of the mandible from the rest of the skull. In the same way, one must dissociate the maxilla above the intercuspidation splint and the mandible (below the splint). The osteotomies are performed using a parallelepiped cutting pattern (Figure $6(\mathrm{~d})$ ). It is interactively placed on the skull model and dimensioned with the manipulation tools provided by Open-Inventor. Those tools are sufficient to obtain a realistic model of surgical cutting off.

Dental Models Integration - The orthognatic diagnosis is subsenquently established (3D position of maxilla, mandible, chin compared to normal) and the surgical simulation is carried out. The $T 1$ matrix is applied to the dissociated dental portions to correct the occlusion on the CT representation. This corrected 


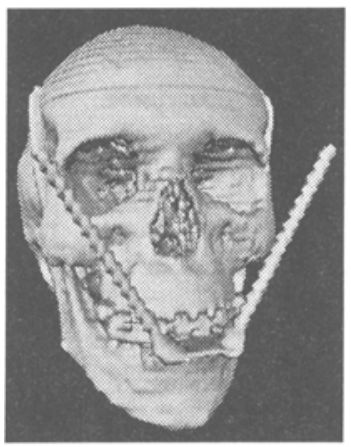

(a)

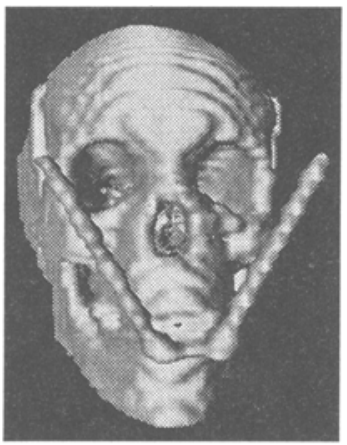

(b)

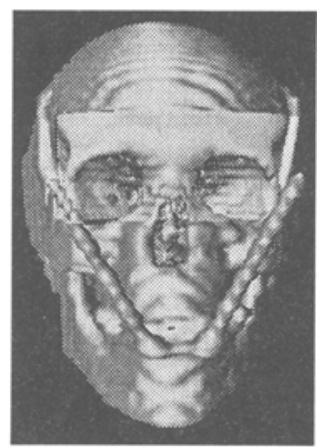

(c)

Fig. 5. (a) High Resolution Model (b) Low Resolution Model (c) Hybrid model. Hybrid model consists of building two models: a high resolution model and a low resolution model reconstructed from averaged CT scan slices. Then, both models are divided into blocks that have the same size. The hybrid model is a combination of these two models divided in blocks. For each cephalometrical point, a default configuration has been defined, but it can also be personnalized by the operator. The low resolution blocks can also be dismissed in order to improve the interactivity and focus on the region of interest.

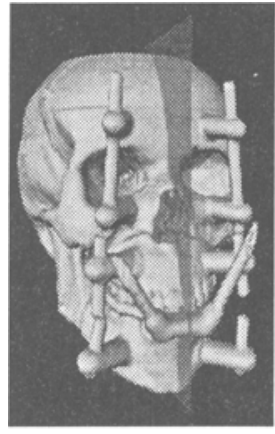

(a)

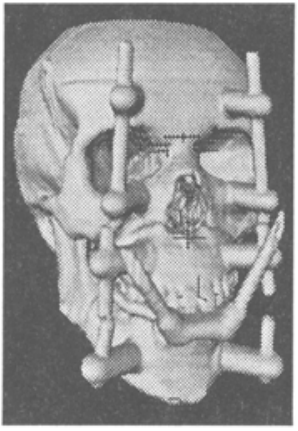

(b)

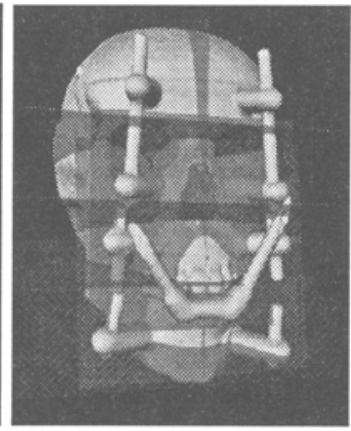

(c)

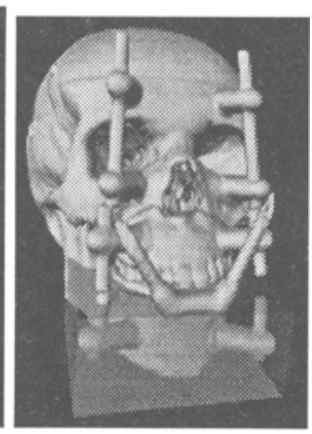

(d)

Fig. 6. 3D Cephalometrical analysis

maxillo-mandibular set is then positionned into the remaining skull respecting the previous orthognathic diagnosis. At this point, the necessity of a genioplasty is evaluated and performed if needed.

Validation of the 3D Cephalometry - The 3D cephalometry used in our simulator has to be validated at two levels. The first level consists of verifying that it is possible to define on the $3 \mathrm{D}$ model reconstructed from CT scan slices the anatomical landmarks (cephalometrical elements). The second level concerns the validation of the $3 \mathrm{D}$ cephalometrical analysis itself and its protocol. The pro- 


\begin{tabular}{|c|c|c|}
\hline Landmark & Error & Norm (mm) \\
\hline$\overline{M_{d}}$ & $-0.853-0.0339-1.508$ & 1.733 \\
\hline$M_{g}$ & $-0.4481 .184-0.148$ & 1.275 \\
\hline$N A$ & $-0.292-0.450-1.299$ & 1.406 \\
\hline$\overline{M e}$ & $-1.607-2.259 \quad 1.769$ & 3.289 \\
\hline$E N A$ & 1.8010 .0270 .007 & 1.802 \\
\hline$I I_{s}$ & $0.2050 .418-2.417$ & 2.461 \\
\hline$C l p_{d}$ & $1.168-0.527-0.902$ & 1.567 \\
\hline$C l p_{g}$ & $1.840-1.976-0.763$ & 2.806 \\
\hline$N p_{d}$ & $-1.5491 .596-1.384$ & 1.533 \\
\hline$N p_{g}$ & $-0.691-0.417-1.303$ & 2.620 \\
\hline Average & & 2.049 \\
\hline Std. Deviation & & 1.606 \\
\hline Minimum & & 1.275 \\
\hline Maximum & & 3.289 \\
\hline
\end{tabular}

Table 1. Results

cedure of validation is realised on a dry skull equipped with its landmarks (tubes) and examinated by computerized tomography. It consists, on the one hand, of digitizing the cephalometric points on the dry skull surface in the optical reference system, and on the other hand, of locating the same points on the $3 \mathrm{D}$ surface model in the CT scan reference system. The $C$ transformation matrix between both referentials is then calculated, either by using the tube-based registration method that has been previously validated, or by using the rigid 3D surface registration algorithm. Therefore, both sets of points are compared and the precision that can be achieved by the operator using the simulator is evaluated.

The results for the 10 chosen anatomical landmarks are shown in Table 1 . Those results are rather satisfactory, since the largest errors $(3.3 \mathrm{~mm})$ correspond to points that are difficult to determine accurately in the real space.

\section{Inferrence}

In order to have a very effective cephalometrical analysis, the determination of the cephalometrical points must be done with accuracy. But this task is very repetitive; it requires all the attention of the operator during a long time. For this reason, we have integrated in the simulator a procedure which defines automatically an initial position for each landmark and their viewpoints. This is done by Inferrence, the process by which the properties of a model are mapped in the patient space using the 3D Octree-splines Transformation. We can use the result of Inferrence between a model and the patient data to display the initial position of the landmarks and to associate viewpoints to each landmark 


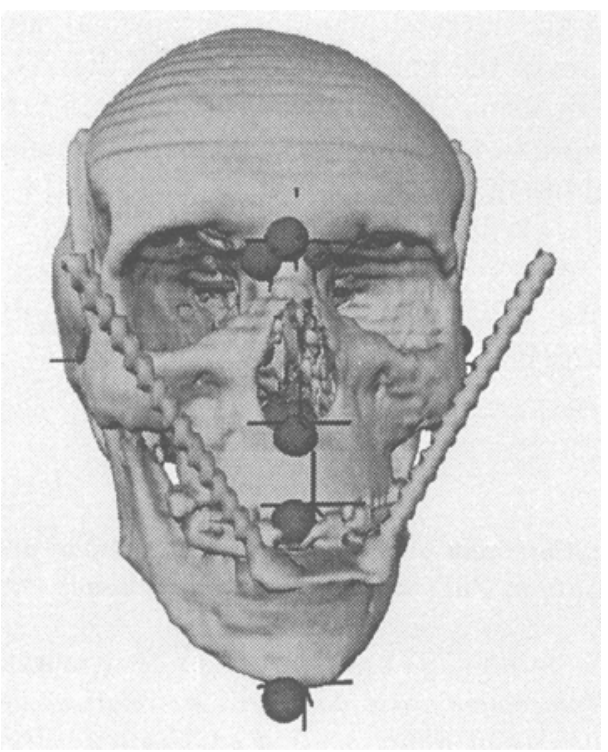

Fig. 7. Illustration of the Inferrence principle. The spheres are landmarks of another model which have been inferred to this model and constitute the initial position for the landmark definition. One can appreciate their relative proximity in comparaison with the final position of the landmarks (crosses).

by inferrence of the model viewpoint. This application is illustrated in Fig. 7 . Initial registration is obtained by alignment of CT coordinate systems (without additional translation or rotation). In this position, the mean distance between landmarks is $21.8 \mathrm{~mm}(\mathrm{~min} / \mathrm{max}=14.2 \mathrm{~mm} / 29.5 \mathrm{~mm})$. After elastic registration and inferrence, the mean distance between landmarks is $5.7 \mathrm{~mm}(\mathrm{~min} / \mathrm{max}$ $=4.3 \mathrm{~mm} / 8.2 \mathrm{~mm}$ ).

\section{Conclusion}

In this paper, we have presented the concepts of the elements necessary for integrated cephalometry analysis, with particular emphasis on the integration of orthodontic information, for optimal and coherent surgical planning. All those elements have been implemented and technically validated with success.

The complete integration and test of the system is now necessary. It will be based on two kinds of experiments. In the first experiment, standard osteotomies and bone displacements can be performed on a dry skull in order to simulate a dysmorphosis. The 3D cephalometry analysis should be able to detect and correct the dysmorphosis. In the second experiment, we will apply the simulation system on the skull of a normal subject. The 3D cephalometry analysis shouldn't detect any dysmorphosis. 
Finally, reproducing the surgical planning during real surgery will be possible by using two elements of the puzzle that we have already validated. First, registration is possible by using the intercuspidation plate fitted with a rigid body, exactly described in section 2. Second, alignment of bone fragments to a predefined position and orientation has been validated on 14 patients, using a passive system for condyle repositionning $[5,3,1]$.

Acknowledgements: this research is financially supported by the european project IGOS HC1026HC.

\section{References}

1. G. Bettega, La Chirurgie orthognathique assistée par ordinateur: de la planification à la réalisation, $\mathrm{PhD}$ thesis, Université Joseph Fourier - Grenoble 1, 23 Novembre 1997.

2. G. Bettega, P. Cinquin, S. Lavallee, and B. Raphael, Dispositif de détermination d'un déplacement entre des positions relatives de 2 moulages dentaires et système de simulation d'intervention en chirurgie orthognatique, patent FR 9616066, (1996).

3. G. Bettega, V. Dessenne, B. Raphael, And P. Cinquin, Computer assisted mandibular condyle positioning in orthognatic surgery, J. Oral Maxillo Facial Surgery, (1996), pp. 553-558.

4. J. DELAIRE, L'analyse architecturale et structurale cranio-faciale (de profil). Principes theoriques. Quelques exemples d'emploi en chirurgie maxillo-faciale., Revue de Stomatologie et de chirurgie Maxillo-faciale, 79 (1978), pp. 1-33.

5. V. Dessenne, G. Bettega, S. Lavallee, P. Cinquin, and B. Raphael, Computer-assisted mandibular condyle positioning in orthognatic surgery, in Second Symposium on Medical Robotics and Computer Assisted Surgery Proc. (MRCAS'95), Baltimore, 1995, Wiley, pp. 215-221.

6. J. M. ET AL., Applications of computer graphics in craniofacial surgery, Clin. Past. Surg., (1986), pp. 441-8.

7. M. V. ET AL., Three-dimensional computer graphics for craniofacial surgical planning and evaluation, in Computer Graphics, vol. 17, 1983, pp. 263-74.

8. E. Keeve, S. Girod, AND B. GIROD, Computer-Aided Craniofacial Surgery, in Computer Assisted Radiology, H. Lemke and al., eds., P.O. Box 211 - $1000 \mathrm{AE}$ Amsterdam - The Nederlands, 1996, Elsevier Science B.V., pp. 757-763.

9. S. Lavallee, R. Szeliski, AND L. BRUnie, Anatomy-based registration of 3- $D$ medical images, range images, $X$-ray projections, 3-D models using Octree-Splines, in Computer Integrated Surgery, R. Taylor, S. Lavallee, G. Burdea, and R. Mosges, eds., MIT Press, Cambridge, MA, 1996, pp. 115-143.

10. LoRENSEN, Marching cube: a High Resolution 3D Surface Construction Algorithm, Computer Graphics, SIGGRAPH'87, 21 (1987), pp. 163-169.

11. R. ROBB AND D. HANSON, The ANALYZE software system for visualization and analysis in surgery simulation, in Computer-integrated surgery, R. e. a. Taylor, ed., MIT Press, Cambridge, MA, 1996, ch. 10. 